BABELAO 4 (2015), p. 1-24

(C) ABELAO (Belgium)

\title{
À propos de deux passages du Grand Hymne à Aton (colonnes 2-3 et 6-7)
}

Par

\section{Claude Obsomer}

Université catholique de Louvain, Université de Namur

L

e Grand Hymne à Aton se lit dans l'embrasure de la porte de la tombe $\mathrm{n}^{\circ} 25 \mathrm{~d}^{\prime}$ Amarna aménagée pour le divin père

Aÿ, un personnage de l'entourage du roi Akhenaton, dont l'épouse Tiyi avait été la nourrice principale de la reine Néfertiti. Contrairement au Petit Hymne à Aton, dont on recense neuf exemplaires répartis dans cinq autres tombes amarniennes, le Grand Hymne ne connait qu'une seule version.

Disposé en treize colonnes au-dessus des figurations d'Aÿ et de son épouse en position d'adoration, le texte fut copié pour la première fois en avril 1883 par Urbain Bouriant, qui le publia dès l'année suivante avec une traduction ${ }^{1}$. Après que le texte eut été partiellement mutilé en 1891 par d'avides marchands d'antiquités, les missions françaises se succédèrent sur le site, pour aboutir en 1903 à une publication incluant le premier fac-similé de l'hymne ${ }^{2}$. En 1908, Norman de Garis Davies publia un second fac-similé,

${ }^{1}$ U. Bouriant, «Deux jours de fouilles à Tell el Amarna », in Mémoires de la Mission archéologique française en Égypte, I, Le Caire, 1884, p. 2-7.

${ }^{2}$ U. Bouriant, G. Legrain, G. JeQUiER, Monuments pour servir à l'étude du culte d'Atonou en Égypte, I (MIFAO, 8), Le Caire, 1903, p. 30-32, pl. XVI-XVII. 
qui fait référence aujourd'hui (fig. 1) ${ }^{3}$. Plus précis que celui de Bouriant, il intègre les lectures de celui-ci pour les portions mutilées du texte. Une telle édition offre l'intérêt de présenter le texte en colonnes en respectant au mieux l'agencement des signes, chose des plus précieuses lorsqu'il s'agit d'examiner les zones qui restent problématiques. L'ouvrage de Maj Sandman, qui, trente ans plus tard, rassemble les principaux textes de l'époque amarnienne $^{4}$, recopie le Grand Hymne en lignes horizontales et continues ; son utilité réside surtout dans les hypothèses de lecture qui sont proposées pour certains passages qui offrent des difficultés.

L'importance du Grand Hymne pour la compréhension de la religion d'Aton lui a valu de faire l'objet de nombreuses traductions, dont les plus intéressantes, citées dans la bibliographie, ont été examinées lors de la rédaction de cet article ${ }^{5}$. On observe que les avis restent partagés sur des questions fondamentales. Ainsi, il n'y a pas de consensus sur l'identité du locuteur : le sujet de $\underline{d} d . f$ « il dit », qui introduit les propos de l'hymne au sommet de la colonne 2, est-il Akhenaton, cité entre Aton et Néfertiti dans le titre de l'hymne qui occupe la totalité de la première colonne, ou bien le divin père $A \ddot{y}$, dont les titres et nom commencent sous cette même colonne? On constate également que le découpage du texte en sections est variable d'un traducteur à l'autre, de même que l'association des unités syntaxiques en vue de composer les phrases complexes. Ainsi, certaines propositions sont associées à ce qui précède par les uns, à ce qui suit par les autres, tandis que d'autres sont considérées tantôt comme des subordonnées, tantôt comme des principales.

Il serait prématuré de proposer ici l'ensemble de la traduction composée lors de la session 2010 de l'ABELAO. Aussi, il ne sera question que de deux passages situés dans la première moitié de l'hymne. Le premier (colonnes 2-3) introduit la description du cycle journalier, où seront évoqués dans l'ordre le coucher du soleil qui produit les ténèbres et son lever qui permet le retour de l'activité. Le second (colonnes 6-7) présente l'action du soleil dans la génération des êtres humains, à laquelle fait suite l'évocation de l'oisillon au sein de sa coquille.

\section{Colonnes 2-3}

Ce passage, dont le texte hiéroglyphique est reproduit à la page suivante, forme un ensemble cohérent puisqu'il est compris entre $\underline{d} d . f$ « il dit» et la circonstancielle qui introduit l'évocation de la nuit: Htp.k $m$ iht imnt(yt) "Quand tu te couches dans l'horizon occidental » (...). L'évocation du jour vient plus loin : $H \underline{d} \underline{d} t^{3}$, wbn.ti $m$ inht « À l'aube, brillant dans l'horizon » (...).

${ }^{3}$ N. DE G. DAVIES, The rock tombs of el-Amarna, VI (ASE, 18), Londres, 1908, pl. XXVII (photographies, pl. I, XLI).

${ }^{4}$ M. SAndman, Texts from the Time of Akhenaten (BiAeg, VIII), Bruxelles, 1938, p. 93-96.

${ }^{5}$ Les références à ces traductions seront données de façon concise (nom de l'auteur), le lecteur étant invité à consulter la liste en fin de cet article. 
Ci-contre, le texte en colonnes extrait de la publication de Davies en 1908. Les pointillés délimitent les zones détruites qui ont été restaurées d'après la copie de Bouriant. Les hachures indiquent les zones dont les signes sont mal conservés.

Ci-dessous, la copie publiée par Sandman. Les zones restaurées d'après Bouriant sont délimitées par les lettres c-d.

Les lettres e-f signalent la variante attestée dans un hymne de la tombe de Mây : sy $n$ s’.k mr.k. Voir N. de G. Davies, The rock tombs of el-Amarna, V (ASE, 17), Londres, 1908 , p. 16, pl. II).

Sandman voit en 3 ylde (remarque g) un terme résultant d'une possible corruption

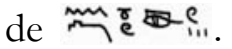

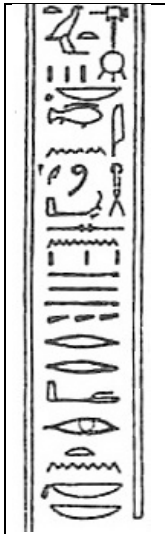

Colonne 3

(haut)

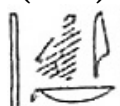

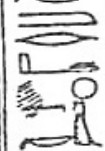

$\stackrel{2}{=}$

5

mo/l/m

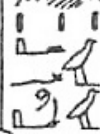

(1)

iा ' 111

$\|=2$

II

"

"I

"

"R 20 "

11'110
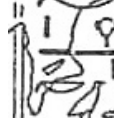

स्या?

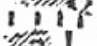

\%.

$3 \%$

$\stackrel{\square}{\stackrel{I}{M} \Omega}$
Colonne 2

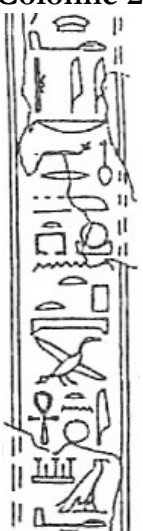

II

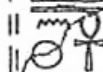

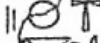
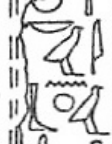

in

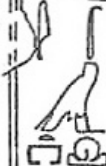

$\triangle \frac{2 f 0}{4}$

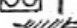

(tillo

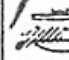
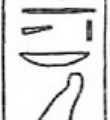

$\ln _{1}$

111

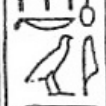

$\underset{\sim}{\mathbb{4}=5}$
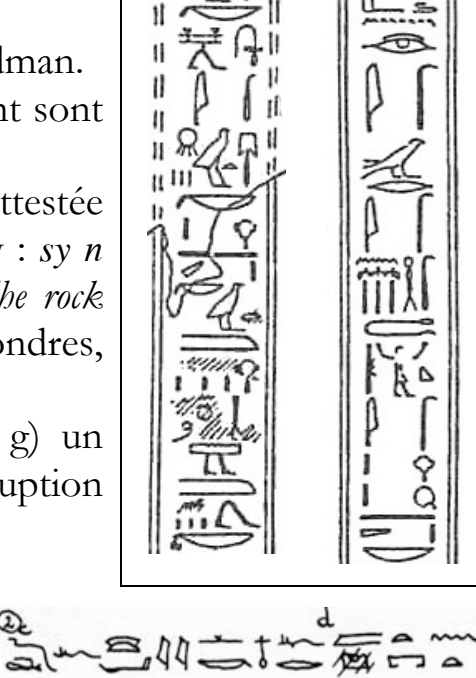

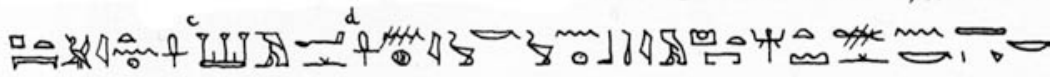

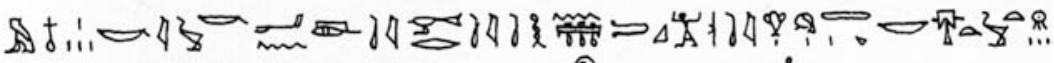

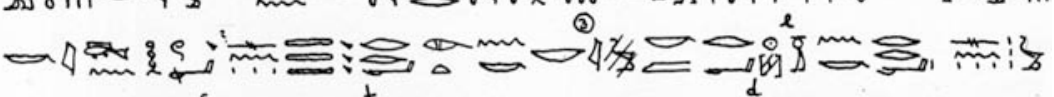

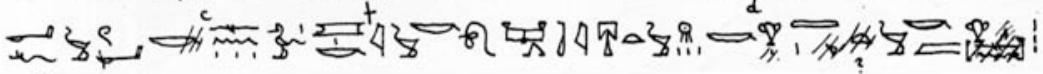

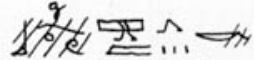


Ce passage comporte douze propositions, verbales ou non verbales, au départ desquelles les traducteurs ont cherché à composer des phrases complexes qui soient douées de sens :

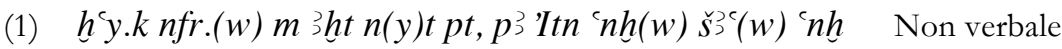

(2) iw.k wbn.ti $m$ sht ỉ bt(y)t

Non verbale en $i w$

(3) mh.n.k t’ nb $m$ nfrw.k

Verbale

(4) iw.k' $n . t i, w r . t i, t h n . t i, k 3 . t i h r-t p t 3 n b$

Non verbale en $i w$

(5) stwt.k, inh.sn t’3w r r- ${ }^{\circ}$ irt.n.k $n b(t)$

Verbale

(6) iw.km $r^{c}$

Non verbale en $i w$

(7) in.kr $r-{ }^{\circ} . s n$

Verbale

(8) $w^{\complement} f w . k s n(n) s^{3} .(k) m r(y) \cdot k$

Verbale

(9) iw.k w3.ti

Non verbale en $i w$

(10) stwt.k hr t?

Non verbale

(11) tw.k m hr. [sn(?)]

Non verbale

(12) $\ldots$ (?) šmwt.k

L'analyse des traductions publiées peut être synthétisée sous la forme du tableau suivant. Les propositions non-verbales introduites par $i w$, importantes dans la discussion qui suivra, sont notées en gras. Les propositions non traduites sont notées par un trait.

\begin{tabular}{|c|c|c|c|c|c|c|c|c|c|c|c|}
\hline Bouriant : & 1 & 23 & 4 & 5 & 6 & 7 & 8 & 9 & 10 & 11 & 12 \\
\hline Erman: & 1 & 23 & 4 & 5 & 6 & 7 & 8 & 9 & 10 & 11 & 12 \\
\hline Davies : & 1 & 23 & 4 & 5 & 6 & 7 & 8 & 9 & 10 & 11 & 12 \\
\hline Scharff : & 1 & 23 & 4 & 5 & 6 & 7 & 8 & 9 & 10 & 11 & 12 \\
\hline Erman-Ranke : & 1 & & 4 & 5 & - & - & - & 9 & 10 & - & - \\
\hline Moret : & 1 & 23 & 4 & 5 & 6 & 7 & 8 & 9 & 10 & - & - \\
\hline Gilbert : & 1 & 23 & 4 & 5 & 6 & 7 & 8 & 9 & 10 & 11 & 12 \\
\hline Wilson: & 1 & 23 & 4 & 5 & 6 & 7 & - & 9 & 10 & 11 & 12 \\
\hline Gardiner : & 1 & 23 & 4 & 5 & 6 & 7 & 8 & 9 & 10 & 11 & 12 \\
\hline Aldred : & 1 & 23 & 4 & 5 & 6 & 7 & 8 & 9 & 10 & 11 & 12 \\
\hline Simpson: & 1 & 23 & 4 & 5 & 6 & 7 & 8 & 9 & 10 & 11 & 12 \\
\hline Assmann : & 1 & 23 & 4 & 5 & 6 & 7 & 8 & 9 & 10 & 11 & 12 \\
\hline Lichtheim : & 1 & 23 & 4 & 5 & 6 & 7 & 8 & 9 & 10 & 11 & 12 \\
\hline Hornung: & 1 & 23 & 4 & 5 & 6 & 7 & 8 & 9 & 10 & 11 & 12 \\
\hline Lalouette : & 1 & 23 & 4 & 5 & 6 & 7 & 8 & 9 & 10 & 11 & 12 \\
\hline Foster : & 1 & 23 & 4 & 5 & 6 & 7 & 8 & 9 & 10 & 11 & 12 \\
\hline Murnane : & 1 & 23 & 4 & 5 & 6 & 7 & 8 & 9 & 10 & 11 & - \\
\hline Grandet : & 1 & 23 & 4 & 5 & 6 & 7 & 8 & 9 & 10 & 11 & $12-$ \\
\hline Mathieu: & 1 & 23 & 4 & 5 & 6 & 7 & 8 & 9 & 10 & 11 & 12 \\
\hline Cannuyer: & 1 & 23 & 4 & 5 & 6 & 7 & 8 & 9 & 10 & 11 & - \\
\hline Laboury: & 1 & 23 & 4 & 5 & 6 & 7 & 8 & 9 & 10 & 11 & 12 \\
\hline
\end{tabular}

Après une analyse aussi complète que possible des choix effectués par les différents traducteurs, je proposerai une nouvelle lecture qui correspond au schéma suivant:

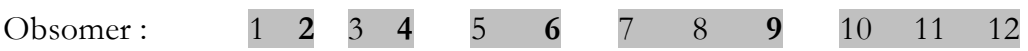




\section{Analyse des traductions existantes}

Pour la majorité des traducteurs, la proposition $\mathbf{n}^{\circ} 1$ constitue une unité indépendante. Elle débute par les termes $h h^{c} y . k n f r$, qui ont fait l'objet de deux analyses différentes :

a. Comme proposition non-verbale :

$H^{\prime} y . k n f r .(w) m$ sht $n(y) t p t .$.

Ton apparition est parfaite dans l'borizon du ciel...

b. Comme proposition verbale :

$H^{c} y . k n f r .(t i) m$ inht $n(y) t p t \ldots$

Tu apparais parfait dans l'borizon du ciel...

Bouriant et Davies firent de $h y . k$ un nom («Splendide est ton lever », "Thy rising is beautiful»), tandis que Erman y vit ensuite une forme verbale de la conjugaison suffixale ( Du erscheinst schön »). Les premiers pensèrent à une proposition non-verbale ayant $h h^{c} y . k$ comme sujet nominal, le second à une proposition verbale. C'est la lecture d'Erman qui fut adoptée par la majorité des traducteurs suivants (Gilbert: "Tu te lèves beau»), mais récemment Mathieu en est revenu à traduire le terme $h^{c} y . k$ comme un nom (« ton apparition »), suivi par Laboury.

Pour trancher la question, il convient d'examiner le second terme de la proposition, $n f r$, qui me semble être de toute évidence un pseudo-participe dont la désinence a été omise dans l'écriture. Dans la première analyse proposée (a), $n f r$ fonctionnerait comme prédicat de la proposition non-verbale à sujet masculin $h h^{c} y . k$ et serait donc à la troisième personne du masculin singulier (forme complète : $n f r . w)$. Dans la seconde (b), $n f r$ se rapporterait au sujet du verbe à la deuxième personne du singulier et devrait lui être accordé (forme complète : $n f r . t i)$. Si l'omission de la désinence du pseudo-participe est tout à fait habituelle dans le cas du $w$ de la troisième personne du masculin singulier, elle est assez rare quand il s'agit du tỉ de la deuxième personne du singulier. On privilégiera donc d'emblée la lecture de $n f r$ comme $n f r .(w)$, en la confortant par les observations: (1) la suite du Grand Hymne note scrupuleusement la désinence tỉ dans la totalité des dix-sept occurrences qu'il comporte de pseudo-participes à la deuxième personne du singulier; (2) toutes les versions du Petit Hymne s'accordent à omettre la désinence de $n f r$ dans la première proposition de cet hymne: $H^{c} y . k / H^{c c} . k n f r .(w), p^{3} \operatorname{Itn}{ }^{`} n h(w)$.

Dans sa proposition initiale, le Petit Hymne atteste généralement la forme géminée $h^{\circledR} . k$, mais on trouve chez Toutou la forme $h^{c} y . k$ qui se lit au début du Grand Hymne. Sans doute convient-il de considérer $h^{c} y$ comme un infinitif (résultant de l'évolution de $h^{c} t$ ) et $h^{c e}$ comme un nom, ce qui revient au même dans la mesure où l'infinitif est substantivé. Le Grand Hymne atteste d'ailleurs la forme $h^{c c} . k$ en tant que nom dans la phrase 'wy.sn $m i 3 w n(y) h^{c c} . k$ " leurs bras sont en adoration de ton apparition » (col. 5); mais c'est la forme brève $h^{c} . k$ qui figure dans la colonne $6: m t n n b$ wn.w $n h^{c} . k$ « tout chemin est accessible en raison de ton apparition ». 
En conclusion, le Grand Hymne commence manifestement par une proposition non-verbale, et, comme dans le Petit Hymne, celleci est suivie de la mention de l'Aton vivant que le locuteur interpelle :

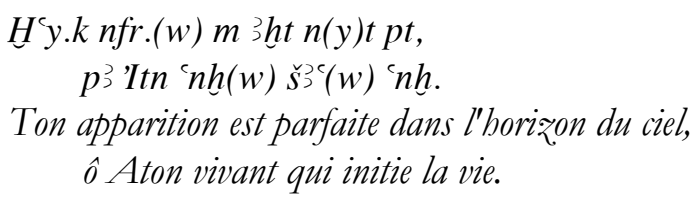

Notons que Pierre Grandet et Christian Cannuyer préfèrent considérer la première proposition de l'hymne comme une subordonnée circonstancielle. Pour Grandet, la principale se situe au niveau de la proposition $n^{\circ} 3:$ : (1) Quand tu poins magnifique à l'horizon du ciel (..), (2) brillant à l'horizon d'Orient, (3) toute terre est par toi emplie de ta beauté ». Pour Cannuyer, il s'agit de la proposition $\mathrm{n}^{\circ} 2$, ce qui lui permet de faire de la proposition $\mathrm{n}^{\circ} 3$ une séquentielle: « (1) Quand tu apparais, parfait, à l'horizon du ciel (..), (2) tu es brillant dans l'horizon oriental (3) et tu as rempli toute la terre de ta perfection ». En réalité, cette approche $n^{\prime}$ est envisageable que dans la mesure où la proposition $n^{\circ} 1$ serait une proposition verbale, ce qui ne semble pas être le cas comme on vient de le montrer ${ }^{6}$.

Tous les traducteurs associent les propositions $n^{\circ} 2$ et 3 au sein d'une même phrase complexe, mais la première (I $I w . k w b n . t i)$ est considérée comme une principale par les uns ${ }^{7}$, comme une subordonnée par les autres ${ }^{8}$ :

(2) 'Iw.k wbn.ti $m$ 3ht ỉ bt(y)t, (3) mh.n.k t’ nb $m$ nfrw.k

a. Tu t'es levé dans l'borizon oriental et tu as rempli toute terre de ta beauté (Laboury)

b. When you have dawned in eastern lightland, you fill every land with your beauty (Lichtheim)

'Iw. $k$ wbn.ti est une proposition non-verbale dont le prédicat est un pseudo-participe (wbn.ti) accordé à un sujet à la deuxième personne du singulier (le pronom suffixe $k$ ). Introduite par l'auxiliaire $i w$, cette proposition peut, en moyen égyptien, correspondre tant à une principale qu'à une subordonnée, parce que son sujet est pronominal. En néo-égyptien, il s'agit d'une subordonnée, car, dans le cas d'une principale, le pronom préfixe $T w . k$ serait utilisé à la place de 'Iw.k. Si certains textes de l'époque amarnienne, comme notamment les stèles-frontières, privilégient l'usage de la syntaxe néo-égyptienne, d'autres offrent un savant mélange des deux états

\footnotetext{
${ }^{6}$ Alan GARDINER avait également choisi d'associer les trois premières propositions en une seule phrase, mais sans pour autant faire de la première une subordonnée : "(1) Thou arisest beauteous in the horizon of heaven (...), (2) when thou didst shine in the eastern horizon, (3) and didst fill every land with thy beauty ».

7 Bouriant, Erman, Gilbert, Hornung, Assmann, Murnane, CANNUYER, LABOURY.

8 Davies, ScharfF, Erman-Ranke, Moret, Wilson, Gardiner, ALDRED, Simpson, LiCHTHEIM, LALOUETTE, Foster, GRANDET, MATHIEU.
} 
de langue et le Grand Hymne à Aton est réputé appartenir à cette seconde catégorie.

Ceux qui traduisent 'I $w$. $k$ wbn.ti comme une principale (a) supposent que le scribe s'exprimait ici en moyen égyptien. Mais le même scribe, voulant exprimer une principale dans la proposition $\mathrm{n}^{\circ} 11$, n'a pas hésité à employer la structure néo-égyptienne : $t w . k$ $m$ hr. [sn (?)] « tu es dans [leur (?)] regard ». Il semble dès lors préférable d'analyser la proposition $\mathrm{n}^{\circ} 2$ comme une subordonnée, sachant que cette solution convient aussi au moyen égyptien. Et il sera dès lors logique d'analyser de même les propositions nonverbales $n^{\circ} 4,6$ et 9 qui sont introduites également par $i w . k$.

Ceux qui voient dans 'Iw.k wbn.ti une subordonnée (b) éprouvent une difficulté quand il s'agit de traduire la proposition $\mathrm{n}^{\circ} 3$, qui serait pour eux la principale. En effet, la forme verbale mh.n.k note un accompli, ce qui donnerait lieu à une traduction comme : «Lorsque tu te lèves / t'es levé / brilles dans l'horizon oriental, tu as rempli toute terre de ta perfection ». Mais la plupart des traducteurs rendent cet accompli par un présent, car l'illumination complète des terres est la conséquence du lever d'Aton, à moins d'attribuer au scribe la volonté d'insister sur le caractère immédiat de son action: "et (déjà) tu as rempli toute terre de ta perfection ». Pour éviter ce problème, Claire Lalouette fait de la forme accomplie mh.n.k une circonstancielle marquant l'antériorité, considérant la proposition $\mathrm{n}^{\circ} 4$ (iw.k ${ }^{\prime} n . t i, \ldots$...) comme la principale : «Tandis que tu apparais dans l'horizon oriental, après avoir rempli le pays tout entier (sic) de ta perfection, tu es beau, grand, étincelant, élevé au-dessus de la terre... ». Mais, dans ce cas, la proposition non-verbale $i w . k$ ' $n . t i$ n'est pas traitée de la même manière que la proposition initiale 'I $w . k$ wbn.ti, alors qu'elle a exactement la même structure. Voilà sans doute ce qui amène Bernard Mathieu à associer les propositions 2 à 5 dans la même phrase, afin que la principale soit la proposition verbale stwt.k inh.sn $t^{\jmath}$ 'w, et $i w . k$ ' $n . t i$ une subordonnée : «(2) Dès que tu t'es levé dans l'horizon oriental, (3) que tu as empli l'univers de ta beauté, (4) radieux, majestueux, éblouissant, bien haut au-dessus de l'univers, (5) tes rayons encerclent les pays jusqu'aux limites de toute ton œuvre ».

En ce qui concerne les propositions $\mathrm{n}^{\circ} 4$ et 5 , les autres traducteurs les traitent le plus souvent comme deux phrases indépendantes, en faisant de $i w . k$ ' $n . t i ̉$ une principale, alors qu'il s'agit à mon sens d'une subordonnée. Ainsi Cannuyer : «(4) Tu es magnifique, grand, étincelant, élevé au-dessus de toute terre. (5) Tes rayons embrassent les pays, jusqu'aux confins de tout ce que tu as créé ».

La proposition $\mathbf{n}^{\circ} \mathbf{6}$, iw. $k m r^{c}$, est de nouveau une proposition non-verbale introduite par $i w . k$, mais dont le prédicat est cette fois un complément prépositionnel composé d'un nom précédé du $m$ dit «d'équivalence ». Elle est suivie des propositions $\mathrm{n}^{\circ} \mathbf{7}$ et $\mathbf{8}$, qui sont deux propositions verbales: (7) in.k $r r^{-}{ }^{\circ} . s n$, (8) $w^{c} f w . k s n$ (n) $s^{3}$.(k) $m r(y) . k$. D'aucuns relèvent un jeu de mots entre $r^{c}$ qui 
désigne le soleil et $r$ - ${ }^{-}$qui signifie les «limites » ou les « confins» (propositions $\mathrm{n}^{\circ} 6$ et 7$)^{9}$.

Rares sont les traducteurs qui considèrent î. $k m r^{c}$ comme une indépendante, en se référant à la grammaire du moyen égyptien ${ }^{10}$. C'est le cas de Lalouette, qui traduit: «(6) Tu es Rê, (7) et tu rapproches leurs extrémités, (8) tu les lies pour ton fils bien aimé ».

La plupart des traducteurs préfèrent voir en iw. $k m r^{c}$ une proposition subordonnée, qu'ils traduisent le plus souvent en la considérant comme une causale : «(6) Parce que tu es le Soleil, (7) tu les atteins jusqu'en leurs confins (8) et tu les lies pour ton fils que tu aimes » (Cannuyer). De plus, certains font de la seconde proposition verbale une circontancielle avec un verbe $w^{c} f w . k$ au prospectif, qui exprime le but ou la conséquence : «(6) Étant Rê, (7) tu atteins leurs limites, (8) de façon à les subjuguer pour ton fils bien aimé » (Mathieu).

De leur côté, Alan Gardiner et Cyril Aldred font se rapporter à ce qui précède la subordonnée ỉ. $k m r^{c}$, comme la raison qui fait que le rayonnement s'étend sur toutes les terres: "(5) Thy rays embrace the lands to the full extent of all that thou hast made, (6) for thou art Re (7) and thou attainest their limits (7) and subdueth them for thy beloved son » (Aldred).

Les propositions $\mathbf{n}^{\circ} \mathbf{9}$ à 12 sont souvent associées deux par deux par les traducteurs. Par prudence, certains s'abstiennent de traduire la proposition $n^{\circ} 12$ dont l'un des mots est difficile à lire.

(9) 'Iw.k w’.tì, (10) stwt.k hrr t’

(11) Tw.k m hr.[sn (?)], (12) ... (?) šmwt.k

$\mathrm{Au}$ sein de la première phrase, les uns font de 'Iw.k w'.ti une principale, ce qui n'est possible qu'en moyen égyptien, les autres une subordonnée. Mais tous conçoivent que l'ensemble vise à énoncer le paradoxe qu'il y a entre l'éloignement du soleil et le fait que ses rayons sont «sur la terre»: «Tu es loin, mais tes rayons sont sur la terre» (Moret, Gilbert) ; «Bien que tu sois loin, tes rayons sont sur terre 》 (Mathieu); «Si éloigné sois-tu, tes rayons sont sur la terre » (Cannuyer).

Comme nous l'avons déjà précisé, la proposition $n^{\circ} 11$ est une proposition non-verbale indépendante ou principale, en raison de l'usage du pronom préfixe $T w . k$ devant le prédicat. Mais les avis sont partagés sur la manière de lire et de traduire ce prédicat. La question dépend de ce qui était noté à gauche du signe du visage de face $(h r)$ dans une zone qui n'a pas été endommagée en 1891 et que Davies a pu examiner dans les mêmes conditions que Bouriant. Mais là où ce dernier voyait un trait et le signe $\mathrm{O} 4$ ( $(\mathbf{Q} \cdot \mathbf{Q}$ ), Davies a identifié trois traits du pluriel sous une lacune ( immor), qui lui semblèrent pouvoir recevoir la notation du pronom suffixe

9 Ainsi Erman, p. 358 n. 3 ; Wilson, p. 370 n. 4 ; ASSMAnN 1975, p. 558 ; LiCHTHEIM, p. 100 n. 2.

${ }^{10}$ ERMAN, DAVIES, SCHARFF, LALOUETTE. 
de la troisième personnel du pluriel (市苗) : Tw.k $m$ hr. [sn] « Thou art in (their) faces ». C'est ce pronom que Sandman restaure dans sa copie du texte. À quoi pourrait se rapporter le pronom restauré par Davies? L'une des phrases de la colonne 5, qui décrit l'activité terrestre qui existe durant le jour, montre que ce pronom pluriel peut se référer au terme $t$ ३ «la terre », pourtant au singulier : «T3 $r$ - $d r . f$, ir.sn k3t.sn «Le pays entier, ils effectuent leurs travaux», c'est-à-dire «Le pays entier effectue ses travaux » au sens de vaquer à ses occupations. Le pronom pluriel désigne ici les différents êtres qui vivent sur la terre et qui sont mentionnés dans la suite. Dans le passage qui nous intéresse, où la proposition précédente s'achève par la mention de la terre (" tes rayons sont sur la terre »), on serait donc amené à comprendre $T w . k m$ hr.[sn] comme "Tu es dans le visage/regard de ses (habitants)». Mais l'espace hachuré dans la copie de Davies est très mince. Il serait envisageable d'y restaurer tout au plus le pronom suffixe de la première personne du pluriel ( 11 . «Tu es dans notre visage/regard». La difficulté est que jamais dans la suite du Grand Hymne, pas plus d'ailleurs que dans le Petit Hymne, le locuteur ne s'associe aux autres humains en utilisant la première personne du pluriel. De son côté, Claire Lalouette omet le pronom suffixe, considérant que le nom serait simplement au pluriel : Tw.k $m$ hrw «Tu es dans les regards». John L. Foster pense même que le terme presque illisible figurant sous le nom pluriel hrw pourrait être un participe fonctionnant comme complément de ce nom: "you shine in the faces of all who turn to follow your journeying ». Mais Foster n'indique pas à quel verbe il pense en traduisant « turn to follow».

Le dernier mot du passage étudié est un nom facile à traduire, šmwt.k «tes cheminements», dont le $\frown t$ du féminin est d'ailleurs encore visible là où Davies nota des hachures (voir fig. 2). Par contre, celui qui précède a fait l'objet d'analyses très diverses, car les éditeurs ne s'accordent ni sur la hauteur de la lacune ni sur les traces qui y seraient encore visibles :

$$
\text { Bouriant: } 0 \text { Davies : } 9 \text { mathe }
$$

Dans sa traduction, «and they watch (?) thy goings », Davies suggère prudemment d'y voir un verbe de perception, idée reprise par Sandman, qui pense à une corruption de du verbe $n w$ « voir » qui apparait au Nouvel Empire comme synonyme des verbes $m 33$ et ptr, auquel serait adjointe la nouvelle forme du pronom suffixe de la troisième personne du pluriel (nw.w « ils voient »). Lalouette suit cette hypothèse, puisqu'elle traduit par «l'on peut contempler ton voyage ». Mathieu reste prudent, tout en liant la proposition à celle qui précède : «(11) tu es devant eux (12) qui contemplent ta course (?)». Spalinger envisage

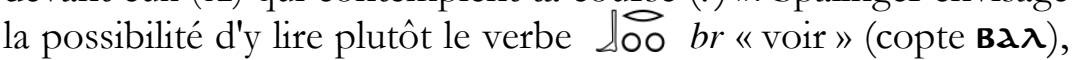
mais ce dernier ne semble attesté qu'à partir de l'époque grecque ${ }^{11}$.

${ }^{11}$ Communication du 18 février 2015. Voir Wb. I, p. 465.6 ; W. VYCICHL, Dictionnaire étymologique de la langue copte, Louvain, 1983, p. 27. 
À l'inverse, de nombreux traducteurs ont considéré que cette proposition était négative, en identifiant la négation $b(w)$ du néoégyptienne dans le signe de la jambe noté par Davies au début du mot, de sorte que l'ensemble de la phrase puisse se comprendre comme suit: " (11) Tu es dans les visages/regards, (12) (mais) on ne voit pas tes cheminements $»^{12}$. La phrase n'est pas sans rappeler celle qui se lit au début de l'Hymne à Amon de Souty et Hor, qui étaient architectes sous Aménophis III (stèle Caire CG 34051): $S t w(t) . k m h r, n r h . t w . s(n)$ "Tes rayons sont sur le visage / dans le regard, (mais) on ne les connait pas ». Aussi plusieurs traducteurs n'hésitent pas à traduire comme suit la proposition $\mathrm{n}^{\circ} 12:$ : $\mathrm{On}$ ne connait pas tes cheminements $»^{13}$. Dans les traces relevées par Davies, Grandet propose même de restaurer une forme passive du verbe $r h$ « connaître » et de translittérer : $B(w) r h .[t] w$ šmwt.k.

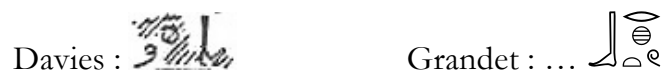

Grandet pousse son analyse plus loin et remet en question le découpage traditionnel des propositions $n^{\circ} 9$ à 12 , en séparant la dernière proposition de celles qui précèdent. Sa traduction est la suivante :

(6) Comme tu es le Soleil, (7) tu atteins leurs confins,

(8) Les plaçant au pouvoir de ton fils bien-aimé,

(9) Lointain (10) dont les rayons sont pourtant sur la terre,

(11) Et de chaque être humain caressent (?) le visage.

(12) Nul ne peut se flatter de connaître ta course,

(13) Quand tu te couches dans l'borizon d'Occident.

Alors qu'il accepte le paradoxe exprimé par l'association des propositions $\mathrm{n}^{\circ} 9$ et 10 , Grandet rejette celui qui est envisagé par les traducteurs qui associent les propositions $\mathrm{n}^{\circ} 11$ et 12 , car "c'est un fait connu que la théologie d'Amarna, fondée sur l'observation de la réalité visible, se refuse à spéculer sur le trajet nocturne inconnu du soleil, contrairement à l'ancienne théologie solaire $»^{14}$. Il choisit alors d'associer la proposition $n^{\circ} 12$ à l'évocation du coucher du soleil qui lui fait suite. Par ailleurs, il refuse de faire de la proposition $n^{\circ} 11$ une proposition non-verbale, mais considère $t w . k$ comme une forme du verbe $t k n$ "s'approcher", qu'il pense retrouver dans un verbe $t w k$ figurant dans une phrase du Petit Hymne attestée seulement chez Apy et Mahou ${ }^{15}$. Mais ce verbe twk, noté avec des déterminatifs, est considéré comme une graphie du verbe $t k$ ` « illuminer » dans le Wörterbuch der Aegyptischen Sprache ${ }^{16}$.

\section{Proposition de traduction}

Comme on l'a souligné à plusieurs reprises, le passage étudié comporte quatre propositions non-verbales introduites par 'Iw.k.

\footnotetext{
12 GARDiner, Simpson, LiCHTHEIM, HORnung, ASSMANN.

13 GILBERT, WILSON, AldRED, GRANDET, LABOURY.

14 GRANDET, p. 139-140.

${ }^{15}$ M. SANDMAN, Texts from the Time of Akhenaten, Bruxelles, 1938, p. 11.

${ }^{16} \mathrm{~Wb}$. V, p. 255 et 333.
} 
Il est tentant de chercher à les traduire toutes de la même façon, soit comme quatre principales (moyen égyptien) ${ }^{17}$, soit comme quatre subordonnées (moyen ou néo-égytien). La seconde solution est préférable, puisque la proposition $n^{\circ} 11$ offre la structure d'une principale néo-égyptienne utilisant le pronom préfixe $T w . k$.

En 1995, William J. Murnane avait opéré ce choix et structuré sa traduction en fonction des quatre propositions en $i w . k$, dans l'idée qu'elles fournissaient autant d'explications quant à la perfection de l'apparition du soleil. Voici l'intégralité de sa traduction ${ }^{18}$ :

(1) Beautifully you appear from the horizon of heaven. O living Aten who initiates life -

(2) For you are risen from the eastern horizon (3) and have filled every land with your beauty;

(4) For you are fair, great, dazzling and high over every land, (5) and your rays enclose the lands to the limit of all you bave made;

(6) For you are $\mathrm{R} e$, (7) having reached their limit (8) and subdued them $<$ for $>$ your beloved son;

(9) For although you are far away, (10) your rays are upon the earth (11) and you are perceived.

Contrairement à Murnane, je pense que ces quatre propositions subordonnées doivent suivre plutôt que précéder la ou les propositions principales dont elles dépendent syntaxiquement, car cela est plus conforme à l'usage observé dans d'autres textes. On aboutit dès lors à un texte en 5 phrases (notées ci-après de $\mathrm{A}$ à $\mathrm{E}$ ), qui est rythmé par le retour des $i w . k$ comme dans une litanie :

A (1) Ton apparition est parfaite dans l'borizon du ciel (3ht $n(y t) p t$ ), $\hat{o}$ Aton vivant qui inities la vie, (2:iw.k) car tu es brillant dans l'borizon oriental (3ht i3bt $(y) t)$.

B (3) C'est de ta perfection que tu as rempli toute terre ( $\left.t^{3} n b\right)$, (4: iw.k) car tu es beau et grand, resplendissant et éminent au-dessus de toute terre $\left(t^{3} n b\right)$.

C (5) Tes rayons embrassent les terres ( $\left.t^{3} w\right)$ jusqu'aux confins $(r-)$ de tout ce que tu as crée, $(6: i w . k)$ car tu es le soleil $(r)$.

D (7) Tu atteins jusqu'à leurs confins ( $r$ - ) (8) afin de les soumettre à ton fils bien-aimé, (9: iw.k) car tu es éloigné.

E (10) Tes rayons sont sur le pays ( $\left.t^{3}\right)$ (11) et tu es dans les regards, (12) (mais) on ne connaît pas (?) tes cheminements.

Dans les quatre premières phrases, les subordonnées introduites par $i w . k$ énoncent les caractères fondamentaux d'Aton : il est brillant dans l'horizon oriental; il est beau, grand, resplendissant et éminent au-dessus de toute terre ; il est le soleil ; il est éloigné. Les principales n'offrent en somme qu'une appréciation complémentaire que le récitant donne sur les actions de l'Aton vivant.

${ }^{17}$ Cette option est celle d'ERMAN 1923.

18 Murnane, p. 113. 
La phrase A gagne à être étudiée en la comparant à la phrase initiale du Petit Hymne, que l'on peut traduire en reproduisant les choix syntaxiques effectués pour le début du Grand Hymne : $H^{`} . k$

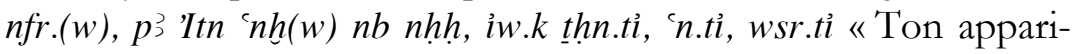
tion est parfaite, ô Aton vivant maitre de l'éternité, car tu es resplendissant, beau et puissant ». La subordonnée consiste ici en un énoncé des qualités de l'Aton vivant similaire à celui de la subordonnée de la phrase B du Grand Hymne, qui emploie d'ailleurs les pseudo-participes thhn.tì et 'n.tì. L'intérêt de la phrase A du Grand Hymne réside dès lors davantage dans les termes qui ne figurent pas au début du Petit Hymne.

Le dieu est décrit comme $\breve{s}^{\jmath^{c}}(w){ }^{c} n h$, une expression totalement inconnue du Petit Hymne qui, pourtant, mentionne à cinq reprises $l^{\prime}$ « Aton vivant». Les traducteurs sont partagés sur le sens à donner à $\breve{s}^{\prime}{ }^{c}(w)$ ' $n h$ dont le verbe $\breve{s}^{\jmath^{c}}$ 《 commencer» se trouve au participe masculin. Les uns pensent qu'Aton est «celui qui a commencé à vivre ", autrement dit celui "qui vit depuis l'origine» (Gilbert) ou « qui vécut le premier» (Mathieu), alors que les autres considèrent que le verbe est transitif direct. Parmi eux, les uns songent à un participe perfectif (Cannuyer: "qui a inauguré la vie »), se référant sans doute à l'acte originel de la création, quand les autres y voient un participe imperfectif, dans l'idée qu'il s'agit d'une création renouvelée chaque matin (Murnane: "who initiates life »). Ma préférence va à la dernière solution, qui s'accorde mieux avec la mention, dans la subordonnée, de l'horizon oriental où l'Aton vivant brille au matin de chaque jour. Un passage du Petit Hymne mentionne d'ailleurs, mais après l'évocation de la nuit, le retour de l'Aton vivant au matin, $m$ ihht ỉ btt $n(y) t p t$ «dans l'horizon oriental du ciel ». Dans la phrase A du Grand Hymne, l'expression se trouve pour ainsi dire scindée en deux, si bien que le terme 3 ht se lit à la fois dans la principale et dans la subordonnée, tout comme $t 3 n b$ «toute terre » sera présente à la fois dans la principale et dans la subordonnée de la phrase B.

Nombreux sont les traducteurs qui rendent la subordonnée $i w . k$ wbn.ti comme une temporelle et certains préfèrent comprendre wbn comme «se lever» plutôt que «briller», en pensant au moment précis où le soleil point à l'Orient. Sans exclure a priori la traduction de iw. $k w b n . t i$ comme «lorsque tu t'es levé », on notera que la temporelle est rendue plusieurs fois par l'aoriste circonstanciel wbn.k. On lit ainsi dans le Petit Hymne, en parlant des êtres qui peuplent la terre: "Ils vivent quand tu brilles pour eux ( $w b n . k$ $n . s n) »$, suivi de «Leurs yeux, quand tu brilles (wbn.k), voient grâce à toi ». Dans le Grand Hymne: "Tes rayons nourrissent toutes les campagnes, quand tu brilles (wbn.k), de sorte qu'elles vivent et poussent pour toi » (colonne 10). Le sens du verbe wbn réclame une étude approfondie, mais "briller» ou «se mettre à briller» peuvent être préférés à «se lever» dans les hymnes où le lever du soleil est rendu par le verbe hi $i$ «apparaittre » ou par le nom $h^{\text {cc }}$ «apparition ». Trois autres passages sont concernés : «Toute fleur est vivante et ce qui pousse sur le sol se développe parce que tu 
brilles ( $n$ wbn.k)» (Petit Hymne); «Tu as créé le ciel lointain pour y briller ( $r$ wbn im.s) et voir tout ce que tu as créé » (Petit Hymne; Grand Hymne, col. 11) ; "Quand tu t'es mis à briller (Wbn.n.k), ils vivent; quand tu te couches (htp.k), ils meurent» (Idem, col. 12).

Le pseudo-participe wbn.ti n'est employé que dans le Grand Hymne, qui offre deux occurrences en plus de celle qui intéresse notre propos. La première se trouve à la colonne 4, dans la phrase qui introduit le passage consacré au lever de l'Aton vivant générateur de l'activité sur terre: "À l'aube, brillant dans l'horizon ( $w b n$.ti $m$ inht) et radieux en tant qu'Aton dans le jour ( $p$ sd.ti $m$ 'Itn $m \mathrm{hrw}$ ), tu repousses les ténèbres et produis tes rayons ». La seconde se lit à la colonne 11 , dans une proposition causale introduite par $i w . k:$ "Tu as créé le ciel lointain pour y briller et voir tout ce que tu as créé, car tu es unique (iw.k $\left.w^{c} . t i\right)$ et brillant en ta forme d'Aton vivant (wbn.ti m 'Itn $n h(w)) »$. Il est clair que dans ces deux cas, le pseudo-participe a une valeur qualitative et non pas résultative : il est brillant en même temps que radieux (ps $\underline{d} . t i)$ ou unique $\left(w^{c} . t i\right)$. Et c'est cette qualité de l'Aton vivant qui peut être énoncée dans la subordonnée de la phrase A, plutôt que le simple fait que son lever s'est opéré.

La phrase B offre en principale une proposition verbale utilisant une forme accomplie sdm.n.f: (3) $m h$ h.n.k $t 3 n b m$ nfrw.k. Les uns la traduisent comme une séquentielle, les autres comme une circonstancielle. Personne jusqu'à présent n'a songé à en faire une principale emphatique, alors qu'elle offre un élément circonstanciel $m n f r w . k$ sur lequel peut porter l'emphase : «C'est de ta perfection que tu as rempli toute terre ». Cette proposition n'est pas sans rappeler un passage du Petit Hymne qui atteste mh.n.k T3wy $m$ mrwt.k, que les traducteurs ont le plus souvent associée à ce qui précède: «L'amour que tu suscites est grand et important, tes rayons sont lumineux vers tous les visages, ta peau est brillante en faisant vivre les cœurs, après/parce que tu as rempli les Deux Terres de ton amour ». Mais elle pourrait tout aussi bien constituer une proposition indépendante. Comme Gilbert, je propose de lui associer plutôt ce qui suit: "C'est de ton amour que tu as rempli les Deux Terres, ô dieu vénérable qui s'est façonné luimême ». Ce choix est légitimé par la présence, dans la colonne 11 du Grand Hymne, d'une forme accomplie sdm.n.f qui est clairement emphatique pour la grande majorité des traducteurs : 'Ir.n.k pt w'.ti $r$ wbn im.s, $r m_{3}^{3}(3)$ iry. $k n b$ « Tu as créé le ciel lointain pour y briller et voir tout ce que tu as créé ». La structure est bien connue en moyen égyptien. L'emphase porte ici sur deux infinitifs de but précédés de la préposition $r$. On notera avec intérêt que la même phrase, dans le Petit Hymne, est introduite par un iw qui permet de la transformer en une proposition autonome moyen-égyptienne, soit également une proposition indépendante.

Notons au passage que, tant dans le Petit Hymne que dans le Grand Hymne, la proposition qui évoque la création du ciel lointain est suivie d'une proposition non-verbale introduite par $i w . k$ qui, suivant la logique de ce qui a été dit plus haut, peut fonctionner 
comme une subordonnée placée derrière la principale. C'est ainsi que procèdent Assmann et Mathieu pour le Petit Hymne, Gardiner, Grandet et Cannuyer pour le Grand Hymne.

Petit Hymne: 'Iw ir.n.k pt w’.t(i) $r$ wbn im.s, $r$ mว iry.k $n b$, iw.k $w^{\complement} . t i$ « Tu as créé le ciel lointain pour y briller et voir tout ce que tu as créé, car tu es unique».

Grand Hymne: 'Ir.n.k pt w’.tì $r$ wbn im.s, $r$ m3(3) iry.k $n b$, iw.k w'.ti

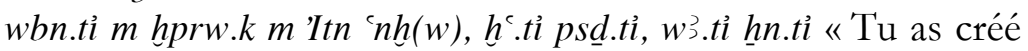
le ciel lointain pour y briller et voir tout ce que tu as créé, car tu es unique et brillant en ta forme d'Aton vivant, et que tu parais éclatant, (à la fois) lointain et proche $»^{19}$.

Alors que, dans le Petit Hymne, la brève subordonnée ỉw.k $w^{c} . t i$, qui offre un jeu de mots probable avec $w^{3} . t(i)$, pourrait aussi se comprendre comme une temporelle (" alors que tu étais seul»), le Grand Hymne offre une subordonnée plus élaborée, causale à mon sens, dont certains termes sont clairement choisis pour trouver un écho dans la principale : wbn.ti « brillant» et $w^{3} . t i \iota$ lointain».

Cette caractéristique, qui semble propre au Grand Hymne, a déjà été observée dans la phrase A de l'introduction, avec la double mention de l'horizon 3ht. Elle se retrouve dans la phrase B qui atteste, tant dans la principale que dans la subordonnée, le terme t3 $n b$ «toute terre». La subordonnée localise un Aton doué de moultes qualités «au-dessus de toute terre », tandis que la principale vise à indiquer qu'il fait bénéficier " toute terre » de ces qualités résumées dans le terme $n f r w$ " perfection ».

Dans la phrase C, deux termes se répondent également, en un jeu de mots entre $r^{-}{ }^{c}$ " les confins» et $r^{c}$ 《le soleil $»^{20}$. C'est la nature même de l'Aton vivant en tant que soleil $r^{\varsigma}$ qui fait que ses rayons agissent jusqu'aux confins $\left(r-{ }^{-}\right)$de la création. Dans la phrase D, c'est son éloignement qui fait qu'il peut atteindre les confins des terres et les soumettre alors au roi d'Égypte, son fils. Ce faisant, il n'est nul besoin d'envisager un paradoxe entre les propositions $\mathrm{n}^{\circ} 9$ et 10 et de rendre le $i w . k$ par une subordonnée initiale qui marquerait une opposition : « (9) Bien que tu sois loin, (10) tes rayons sont sur terre » (Mathieu).

La phrase E, dernière phrase du passage étudié, mentionne la terre au singulier $\left(t^{3}\right)$, si bien qu'il est probable que l'énoncé concerne spécifiquement l'Égypte, et plus précisément la perception que ses habitants peuvent avoir de l'Aton vivant. Son interprétation reste délicate, car le texte ne précise pas comment il convient de concevoir les «cheminements » (šmwt) de l'Aton vivant. S'agitil nécessairement de son cheminement nocturne, comme le pense Grandet? En évoquant la nuit, lorsque « le pays ( $\left.t_{3}^{3}\right)$ est dans les

19 GARDINER : «Thou hast made the sky distant to shine in it and to see all that thou hast made, being alone and shining in thy various forms as the living Aten, appearing gloriously and gleaming, being both distant and near ».

${ }^{20}$ Jusqu'à présent, ce jeu de mots avait été relevé seulement entre le nom $r^{c}$ et le $r$ - ${ }^{`}$ qui figure dans la proposition $n^{\circ} 7$. Voir ci-dessus, p. 8, note 9. 
ténèbres en état de mort», le Grand Hymne se contente d'indiquer que « celui qui les a créés repose dans son horizon 3̧ht » et l'on sait que la conception d'un parcours souterrain tel que le décrit le Livre de l'Amdonat est écartée à l'époque amarnienne. Pourrait-il s'agir, au contraire, du cheminement des rayons durant la journée? Pourrait-il s'agir d'une réflexion à propos de la lumière produite par l'Aton vivant, véhiculée par des rayons invisibles à l'œil nu, mais que l'iconographie, capable de figurer l'invisible, a néanmoins réussi à matérialiser? N'est-ce pas le même constat qui serait à la base de l'énoncé qu'on lisait dans l'Hymne à Amon de Souty et Hor: Stw $(t) . k m h r, n$ rh.tw.s(n) «Tes rayons sont sur le visage / dans le regard, (mais) on ne les connait pas »?

\section{La copie de la tombe de Mây}

Le début du Grand Hymne est noté dans l'entrée de la tombe $\mathrm{n}^{\circ} 14$ du général Mây, sous le relief montrant l'adoration du soleil par la famille royale, dans les premières colonnes d'un hymne composé pour ce Mây ${ }^{21}$. Voici l'édition publiée par Davies :

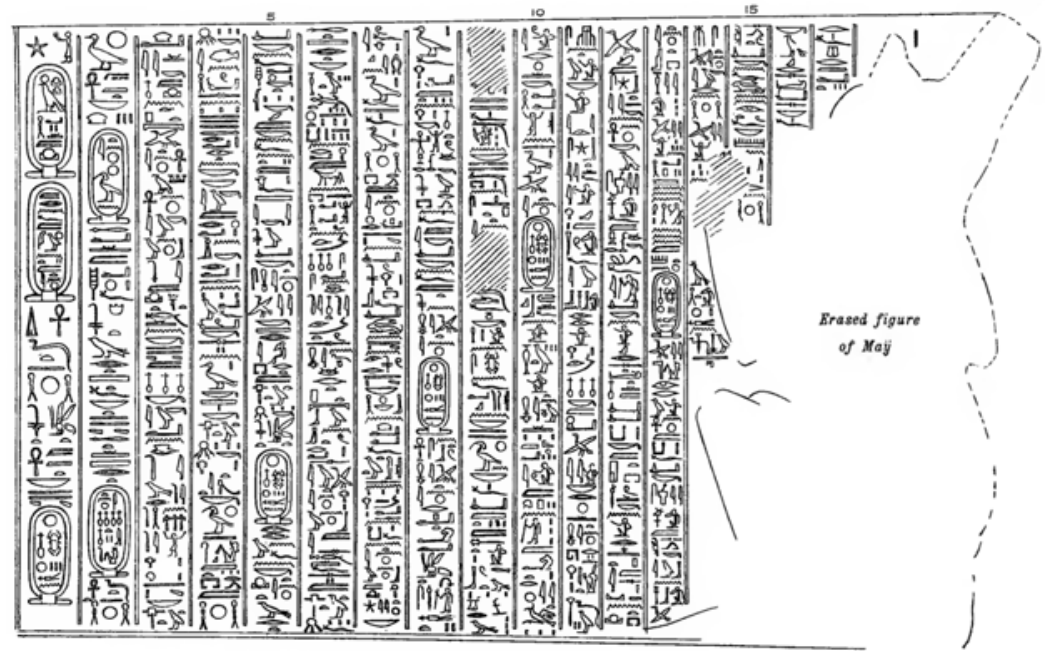

Les deux premières colonnes associent, après le terme initial $d w$ ? « adorer » ou « adoration », non seulement les noms d'Aton et d'Akhenaton, mais aussi celui de Néfertiti, comme c'était le cas pour le Grand Hymne de la tombe d'Aÿ. Mais l'hymne lui-même n'est pas introduit par le verbe $\underline{d} d . f$ « il dit ». Ce sont les colonnes 3 et 4 qui reproduisent partiellement le passage qui vient d'être étudié, à l'exception de la proposition $\mathrm{n}^{\circ} 9$.

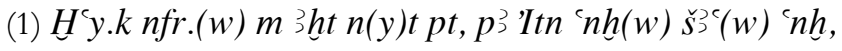

(2) iw.k wbn.ti m int ỉbt(y)t.

(3) Mh.n.k t3 nb $m$ nfrw.k,

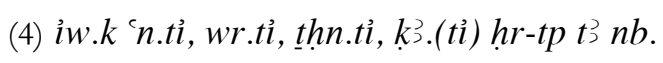

(5) Stwt.k, inh.sn t’3w (r) r- 'irt.n.k nb(t),

(6) $i w . k m r$.

(7) 'In.k (r) $r{ }^{-}{ }^{c} . s n(8) w^{c} f w . k$ sy $n s^{3} . k m r(y) . k$.

${ }^{21}$ N. DE G. DAviES, The rock tombs of el-Amarna, V (ASE, 17), Londres, 1908, p. 16, pl. II, XIX (photographie); M. SANDMAN, Texts from the Time of Akhenaten (BiAeg, VIII), Bruxelles, 1938, p. 59-60. 
Le texte exploite ensuite la mention d'Akhenaton comme «ton fils bien-aimé » pour indiquer les dons que le roi a reçus du dieu, en commençant par la phrase suivante: Stwt.k hr tit.k $3 h t^{22}, h k^{3}$ $M{ }^{`} t \operatorname{pr}(w) m n h h$ «Tes rayons sont sur ton image bénéfique, le souverain de Maât qui procède de l'éternité ». L'expression tit 3ht n'est pas sans évoquer le nom même d'Akhenaton, 3̌h $n$ 'Itn «Bénéfique à Aton ». Cette volonté d'orienter le discours vers la personne du roi peut expliquer en soi l'omission de la proposition $\mathrm{n}^{\circ} 9$ (iw. $\left.k w^{3} . t^{i}\right)$ lors de l'adaptation du texte. La suite mentionnera la création d'Akhetaton par le roi pour le service du dieu.

\section{Colonnes 6-7}

Le second passage à examiner est le suivant :

À droite, le texte en colonnes d'après la publication de Davies en 1908.

Ci-dessous, la copie de Sandman.

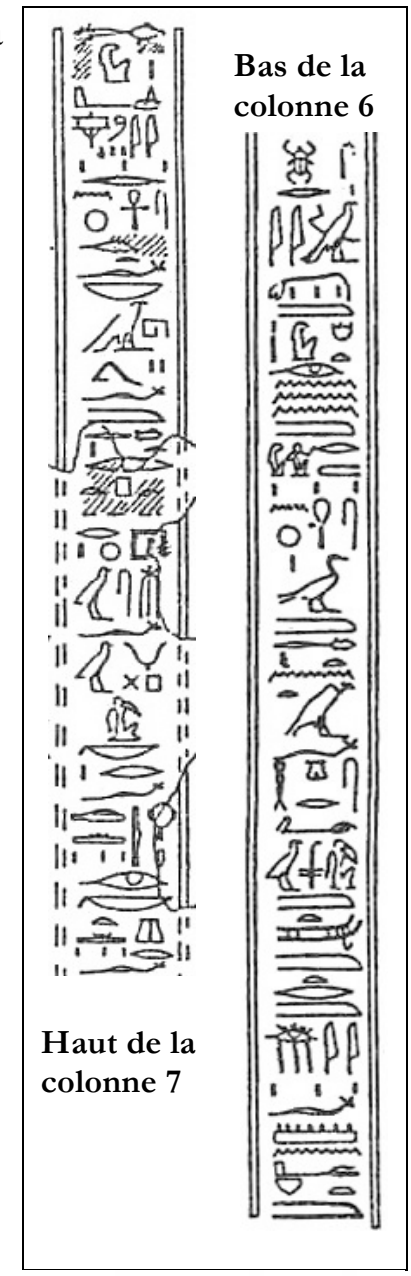

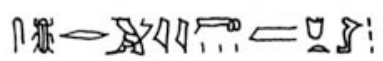

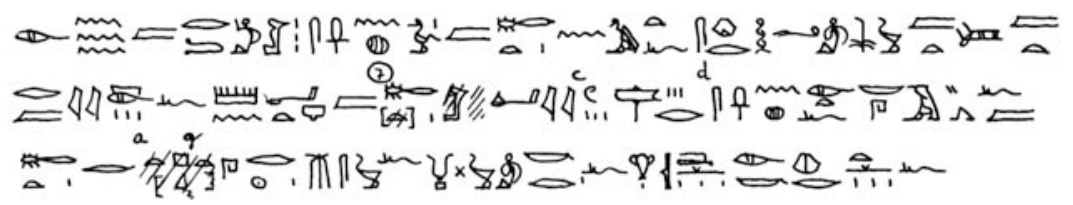

Ce passage comporte un certain nombre de jeux de mots qui n'ont pas été notés par les commentateurs. La translittération qui suit offre une nouveauté à la fin de la section 6 :

${ }^{22}$ La désinence $-t$ du féminin semble bien figurer dans l'inscription, d'après une photographie prise en février 2015 par Nicolas Gauthier. 
(1) $\operatorname{Shpr}(w)$ mзyw $m$ ḥmwt, (2) ir(w) $m w w m r m t$,

(3) $\sinh (w) s^{3} m \underline{h} t n(y t) m w t . f$, (4) $\operatorname{sgrh}(w) s w m$ tmt rmy (t).f,

(5) $m n^{c} t m$ ht , (6) dy țisw r s'nh ir $r(w)$ it $n b$,

(7) $h$ 3.f $m$ ht r tpt, hrw msw(t).f, (8) wp.k r(3).f hrr-kd, ir.k hrt.f.

Bouriant et Davies avaient associé le début de ce passage à la phrase qui précède : "Tes rayons, qui pénètrent jusqu'au sein de la Grande Verte, (1) font féconder les femmes, (2) forment la semence dans les hommes (3) et animent l'enfant dans le sein de sa mère » (Bouriant). Mais cette hypothèse fut vite écartée, les autres traducteurs préférant associer cette phrase à celle qui précède en concluant ainsi la description de l'activité générée par le soleil durant le jour: Rmw hr itrw hrr tft $n$ ḥr.k, stwt.k $m$ - $\underline{h} n w$ w’ $\underline{d}$-wr «Les poissons du fleuve s'agitent devant toi, (car) tes rayons sont au sein de ouadj-our ».

Les quatre premières formes verbales (shpr, ir, $s^{c} n h$ et $\left.s g r h\right)$ sont analysées diversement par les traducteurs. La plupart les considèrent comme des participes actifs masculins, énoncés de façon autonome, tout en se rapportant au dieu à qui l'on s'adresse. Aussi ajoutent-ils une référence à la deuxième personne du singulier, qui serait sous-entendue. Voici par exemple la traduction de Mathieu :

(1) Toi qui produis les germes chez les femmes,

(2) et qui changes la semence en être bumain,

(3) Qui vivifies l'enfant dans le sein de sa mère,

(4) qui l'apaises et taris ses larmes,

(5) Nourricier dans le sein, (6) dispensateur du souffle, pour vivifier toute ton cuvre!

(7) Lorsqu'il sort du sein pour respirer, au jour de sa naissance,

(8) tu ouvres grand sa bouche pour subvenir à ses besoins.

Certains n'hésitent pas à traduire comme si les premiers verbes attestaient effectivement un pronom suffixe $e^{23}:$ « (1) Tu rends les femmes fécondes (2) et crées la semence chez les hommes, (3) faisant vivre le fils dans le sein de sa mère (...)» (Lalouette).

De son côté, Grandet préfère voir des formes accomplies, au passif, dans les quatre premiers verbes, ce qui, selon Cannuyer, ne s'impose pas :

(1) Voici que dans les femmes l'embryon est formé,

(2) Voici que dans les hommes est créé la semence,

(3) Et l'enfant animé dans le sein de sa mère,

(4) Apaisé par ce qui lui fait cesser ses pleurs.

Bien entendu, on pourrait envisager de comprendre ces formes verbales comme des impératifs : «Produis..., change..., vivifie..., apaise... ». Mais l'impératif n'est pas attesté par ailleurs lorsqu'on s'adresse à Aton. Il convient dès lors d'en revenir à l'analyse la plus courante et de voir dans ces verbes des participes masculins. J'irais même plus loin, en considérant l'ensemble du passage examiné comme une seule et même phrase, car les deux dernières

${ }^{23}$ ERMAN-RANKE, GilBERT, Simpson, LALOUETTE. 
formes verbales offrent un sujet à la deuxième personne : (8) wp.k $r(3) . f h r-k d$, ir.k hrt.f « tu lui ouvres la bouche entièrement et tu pourvois à ses besoins » ( $c f r$ Cannuyer). La forme wp.k constituera dès lors le verbe principal, tandis que ir.k pourra, éventuellement, passer pour un prospectif circonstanciel exprimant le but, voire la conséquence. Voici la traduction que je propose :

(1) (Toi) qui produis les semences (mзyw) dans les femmes ( $h m w t)$,

(2) qui transformes le fluide ( $m w$ ) en êtres bumains ( $r m \underline{t})$,

(3) (toi) qui vivifies le fils dans le ventre de sa mère (mwt.f),

(4) qui l'apaises en cessant ses larmes (rmy $(t) \cdot f)$,

(5) (toi qui es) une nourrice dans la gent féminine,

(6) qui donnes le souffle pour vivifier tout être qu'un père engendre,

(7) lorsque celui-ci sort du ventre pour respirer, le jour de sa naissance,

(8) tu lui ouvres sa bouche entièrement et tu pourvois à ses besoins.

Les deux premières lignes établissent un parallèle entre $m 33 y w$ et $m w$. Le second terme est connu pour désigner le sperme masculin aussi bien que l'eau, tandis que le premier, qui est noté au pluriel, semble être un hapax ${ }^{24}$. Les uns y voient les semences que sont les spermatozoïdes, les autres pensent à des embryons ou fœtus qui croissent dans les ventres féminins. Comme me l'a fait remarquer Didier Devauchelle, le déterminatif du phallus est à prendre en considération : il plaide sans doute pour la première solution ${ }^{25}$.

Contrairement à ce que laissent entendre certaines traductions, qui rendent par «les hommes» le terme rmt, il ne semble pas y avoir d'opposition entre ce dernier et le terme hmwt «les femmes » de la première ligne car rmt désigne l'ensemble des êtres humains comme le confirme le déterminatif. Par contre, il est utile de rappocher les termes qui concluent les lignes 3 et 4 de ceux qui concluent les lignes 1 et 2 , puisqu'ils offrent une homophonie évidente. Ces couples de mots ainsi formés sont porteurs de sens, car la mère $(m w t)$ fait partie des femmes $(h m w t)$, tandis que l'association des larmes (rmyt) et des êtres humains ( $r m t$ ) constitue un jeu de mot bien connu des textes égyptiens ${ }^{26}$. Si les trois premières lignes décrivent le développement du fœus, qui est désigné par le mot $s$ « fils » par référence au terme mwt.f «sa mère » de la même ligne, la quatrième semble, par contre, un peu artificielle. La volonté d'utiliser à tout prix le terme $r m y(t)$ a dû pousser l'auteur à imaginer que c'était le dieu qui évitait au fotus de pleurer avant sa naissance. Ce dont ce foetus a surtout besoin, c'est de respirer et de se nourrir. Tel est l'objet des lignes 5 et 6 .

La ligne 5 identifie Aton à une nourrice - ce qu'avait d'ailleurs été l'épouse d'Aÿ, Tiyi, pour Néfertiti -, et il est une nourrice $m \underline{h} t$. Le nom $h t$ été compris comme le ventre de la mère, le sein mater-

${ }^{24} \mathrm{~Wb}$. II, p. 12.10

25 Suivant $A$ Lex, $\mathrm{n}^{\circ}$ 79.1103, on lit peut-être chez Ramsès II une graphie féminine de ce nom avec cette fois le déterminatif de l'œuf: «Les couronnes ont été assemblées pour toi, alors que tu n'étais que semence (myt ou m³yt?) dans le ventre de ta mère Isis » (KRI II, 197.11).

26 " J'ai fait venir à l'existence les dieux de ma sueur, (mais) les hommes des larmes de mon œil » (Textes des Cercueils, 1130). 
nel. Grandet estime que ce nom se trouve ici noté au pluriel ( $\underline{h} w t$ «les ventres »), au contraire des lignes 3 et 7 qui l'attestent au singulier. La copie de Davies présente, en effet, des hachures à la fin du mot où l'on peut effectivement penser à y restituer trois traits verticaux disposés les uns sur les autres (haut de la colonne 7, voir aussi la fig. 3). En outre, à la différence des deux autres notations de $h t$, le nom est accompagné du déterminatif de la femme (signe B 1), ce qui amène peut-être Laboury à traduire «le ventre des femmes ». Mais la graphie du mot permet aussi de penser à une notation du nom collectif féminin $\underline{h} t^{27}$, connu par ailleurs pour désigner une corporation divine, un corps d'armée ou une génération humaine ${ }^{28}$. Dans ce cas, comme le déterminatif de l'homme est omis et que seul est noté celui de la femme, il peut très bien s'agir d'une désignation de la gent féminine dans son ensemble.

À la fin de la ligne 6, Aton donnerait le souffle $r s^{c} n h$ irt.f $n b(t)$ "pour vivifier tout ce qu'il crée (sc. sa création)», si l'on suit les traductions antérieures. L'utilisation du suffixe de la troisième personne a de quoi étonner si l'on est censé s'adresser au dieu. Mais l'on pourrait lever cette difficulté en considérant que le participe $d y$ est substantivé et fonctionne en parallèle au nom $m n^{c} t$ : " (toi qui es) une nourrice (...) et celui qui donne le souffle pour vivifier tout ce qu'il crée ». Toutefois deux observations doivent être faites à ce stade. La première est que, dans les hymnes amarniens, c'est la forme relative masculine, iry. $k$ ou iry.k $n b$, qui est régulièrement employée pour désigner la création : le recours à une forme neutre, issue de l'usage du moyen égyptien, n'est attesté qu'une fois, à l'accompli, dans la colonne 2 (irt.n.k $n b(t))$. La seconde est que toutes les lignes de ce passage évoquent très précisément la génération d'un être humain, la ligne 6 étant la seule qui aurait une portée tout à fait générale, envisageant l'ensemble des choses créées. Aussi, il me semble possible d'envisager une autre lecture, sachant que le nom it «père» peut s'écrire Empire : $r$ s'nh ir(w) it $n b$ "pour vivifier tout (être) qu'un père engendre ». Cette lecture permet aux lignes 5 et 6 d'associer une allusion aux femmes et aux hommes, tout comme le faisaient les deux premières lignes. De plus, le «père » serait présent à la ligne 6 , tandis que la ligne 3 mentionnait la «mère ». En analysant de cette façon la ligne 6 , on obtient en outre l'équivalent d'un nom masculin, "tout être qu'un père engendre », auquel peuvent se référer les quatre pronoms suffixes masculins attestés aux deux dernières lignes, qui évoquent la naissance de l'enfant. Nul besoin dans ce cas de remonter à la ligne 3 pour y trouver le nom masculin « fils ».

Le passage est suivi de la description de l'oisillon $\underline{t}$, que l'on comparera évidemment à ce qui vient d'être dit de l'enfant. Aux six premières lignes décrivant l'enfant avant sa naissance, auquel le dieu donne le souffle de vie, correspond la première phrase :

${ }^{27}$ Les trois traits notent aussi bien un collectif singulier qu'un pluriel.

${ }^{28}$ Notamment $W b$. III, p. 357-358 ; ALex, n 77.3216, 78.3175, 79.2301 ; R. HANNIG, Ägyptisches Wörterbuch, II, Wiesbaden, 2006, p. 1972-1973. 
Iw ţ $m$ swht $m d w(w) m i n r$, di.k n.f tisw m-hnw.s $r s^{`} n h . f$.

L'oisillon dans l'cuf "parlant» dans la coquille, tu lui donnes le souffle à l'intérieur de celui-ci pour le vivifier.

La seconde phrase insiste sur la maturité ${ }^{29}$ qui est celle de l'oisillon, à l'inverse du nouveau-né humain, dès le moment de l'éclosion :

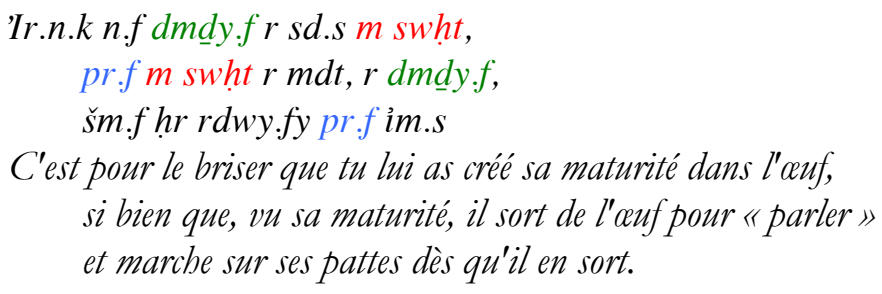

\section{En guise de conclusion}

Les hymnes à Aton comptent parmi des textes fondamentaux pour la compréhension de la religion d'Akhenaton. Conservés dans les tombes privées d'Amarna, ces hymnes, dont la rédaction est souvent attribuée au roi lui-même, étaient sans doute récités lors des célébrations au temple. La traduction proposée ici pour l'introduction du Grand Hymne (colonnes 2-3) va dans ce sens, car elle présente une série d'énoncés qui s'achèvent par une même proposition en $i w . k$ comme dans une litanie.

Le Grand Hymne n'est attesté intrégalement que dans la tombe d'Aÿ, mais la tombe de Mây en présente néanmoins le début. L'expression $n s^{3} . k m r(y) . k$ "à ton fils bien-aimée », qui figure dans la proposition $\mathrm{n}^{\circ} 8$ de l'introduction de l'hymne, est notée chez Aÿ avec l'omission de deux signes importants, qui sont présents chez Mây. Il semble donc évident que le Grand Hymne n'est pas une composition propre à $A \ddot{y}$, mais que ce dernier a fait recopier dans sa tombe un texte connu par ailleurs et dont Mây aurait choisi de ne copier que le début.

Sur la question de la filiation des deux hymnes, Mathieu pense que le Petit Hymne est le prototype du Grand Hymne ${ }^{30}$. Cette idée est séduisante, bien qu'elle reste à démontrer. On pourrait penser qu'il y aurait eu une évolution dans le texte de l'hymne récité au temple d'Aton, comme il y a eu une évolution dans la façon de figurer le dieu et de noter son nom. Toutefois, on observe que certaines copies du Petit Hymne se trouvent dans des tombes qui attestent la seconde version du nom d'Aton (Any, Mérirê et Mahou), alors que les tombes d'Aÿ et de Mây attestent l'emploi de la première version du nom divin, comme les tombes d'Apy et de Toutou qui offrent aussi le Petit Hymne. Il semble donc que la composition du Grand Hymne, si elle est postérieure à la rédaction du Petit Hymne, n'a pas supplanté l'usage de ce dernier. Peut-être le Grand Hymne était-il réservé à un certain type de célébration.

\footnotetext{
${ }^{29}$ Je reprends de Cannuyer la traduction de $d m \underline{d} y$ par « maturité ».

30 Mathieu 1999, p. 36.
} 


\section{Traductions (dans l'ordre chronologique)}

U. BOURIANT, « Deux jours de fouilles à Tell el Amarna », in Mémoires de la Mission archéologique française en Égypte, I, Le Caire, 1884, p. 2-7 ;

A. ERMAn, A Handbook of Egyptian Religion, Londres, 1907, p. 6466 (trad. F.L. GRIFFITH à partir de l'édition allemande de 1904) ; A. Erman, Die Literatur der Aegypter, Leipzig, 1923, p. 358-362;

N. DE G. DAviES, The Rock Tombs of el-Amarna, VI, Londres, 1908, p. 29-31;

A. SCHARFF, Aegyptische Sonnenlieder, Berlin, 1922, p. 61-66;

A. Erman, H. Ranke, Aegypten und aegyptisches Leben im Altertum, Tübingen, 1923, p. 462-464 ;

A. Moret, Le Nil et la civilisation égyptienne, $2^{\mathrm{e}}$ éd., Paris, 1937, p. 378-379;

P. GILBERT, La poésie égyptienne, Bruxelles, 1943, p. $34-39$ ( $2^{\mathrm{e}}$ éd., Bruxelles, 1949, p. 36-40);

J.A. WILSON, «Egyptian Hymns and Prayers », in J. Pritchard (éd.), ANET, Princeton, 1950, p. 369-371;

A.H. Gardiner, Egypt of the Pharaohs, Oxford, 1961, p. 225-227 ;

C. Aldred, Akhenaten, Pharaoh of Egypt: a New Study, Londres, 1968, p. 187-189 (C. ALDRED, Akhenaton, le pharaon mystique, Paris, 1969, p. 185-187) ;

W.K. Simpson, The Literature of Ancient Egypt, New Haven, Londres, 1973, p. 289-295 ;

J. Assmann, Ägyptische Hymnen und Gebete, Zürich, Munich, 1975, p. 215-221 ; « Ägyptische Hymnen und Gebete », in Lieder und Gebete, II (TUAT, II.๑), Gütersloh, 1991, p. 848-853 ;

M. LiCHTHEIM, Ancient Egyptian Literature, II, Berkeley, Los Angeles, Londres, 1976, p. 96-100 ;

E. Hornung, Meisterwerke altägyptischer Dichtung, $2^{\mathrm{e}}$ éd., Zürich, 1979, p. 69-73 (idem in H.A. Schlögl, Echnaton-Tutanchamun, Wiesbaden, 1983, p. 78-80);

Cl. LALOUETTE, Textes sacrés et textes profanes de l'ancienne Égypte II, 1987, p. 126-129;

J.L. FOSTER, Echoes of Egyptian Voices, Norman, Londres, 1992, p. $5-10$;

W.J. Murnane, Texts from the Amarna Period in Egypt, Atlanta, 1995, p. 112-116;

P. Grandet, Hymnes de la religion d'Aton, Paris, 1995, p. 97-119, p. 137-152;

B. Mathieu, «Le Grand Hymne à Aton », in Égypte, Afrique et Orient 1999, p. 35-44 (idem in M. GABOLDE, Akhénaton. Du mystère à la lumière, Paris, 2005, p. 98-99) ; B. MATHIEU, «Le Grand Hymne à Aton ", in Akhénaton et l'époque amarnienne, Paris, 2005, p. 105-116;

Ch. CANNUYER, «Questions sur la religion d'Akhénaton et son prétendu "monothéisme" », Mélanges de science religieuse, 59, 2002, p. 23-82;

D. Laboury, Akhénaton, Paris, 2010, p. 449-451. 


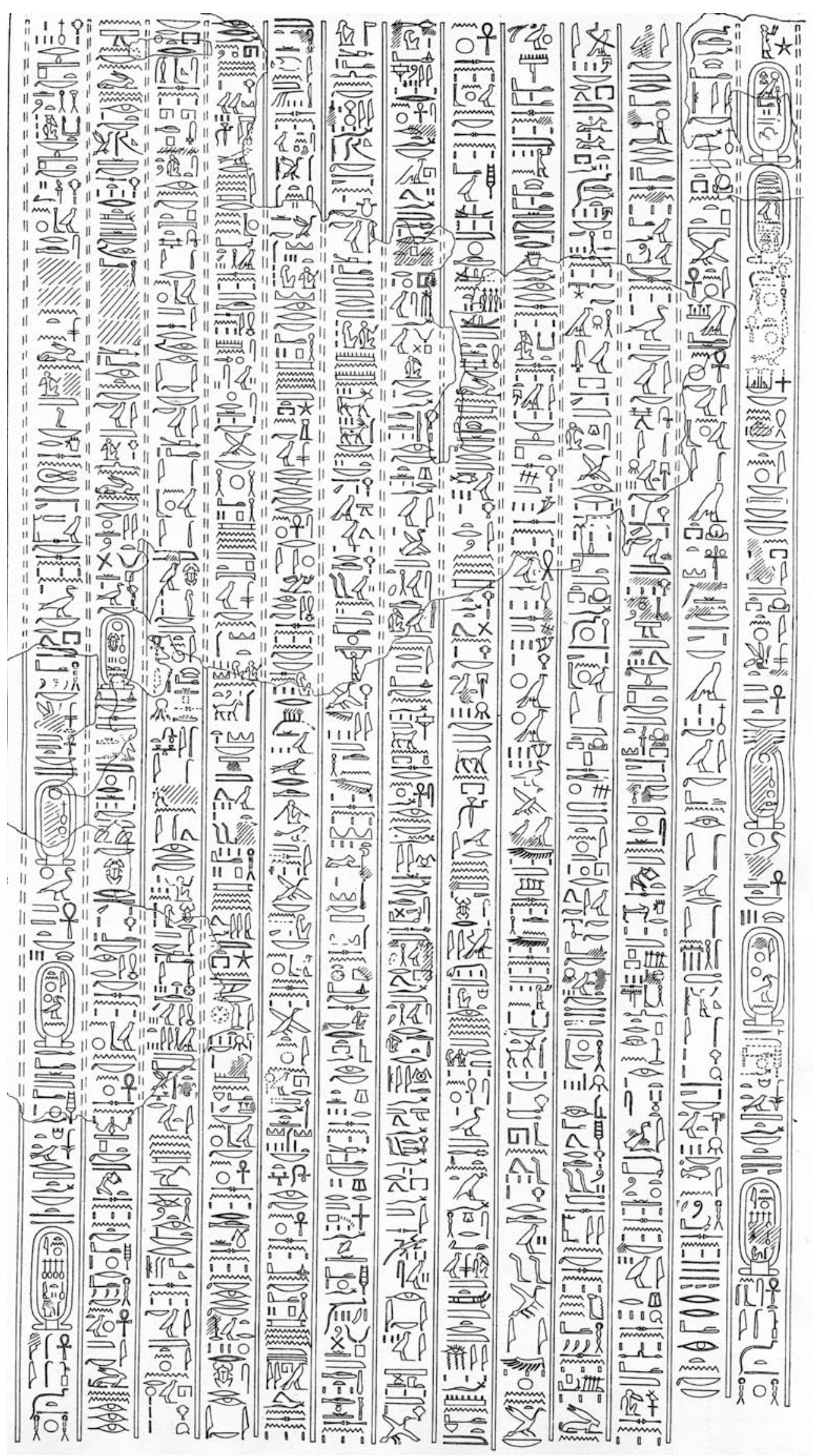

Fig. 1. N. DE G. DAVIES, The rock tombs of el-Amarna, VI (ASE, 18), Londres, 1908, pl. XXVII 


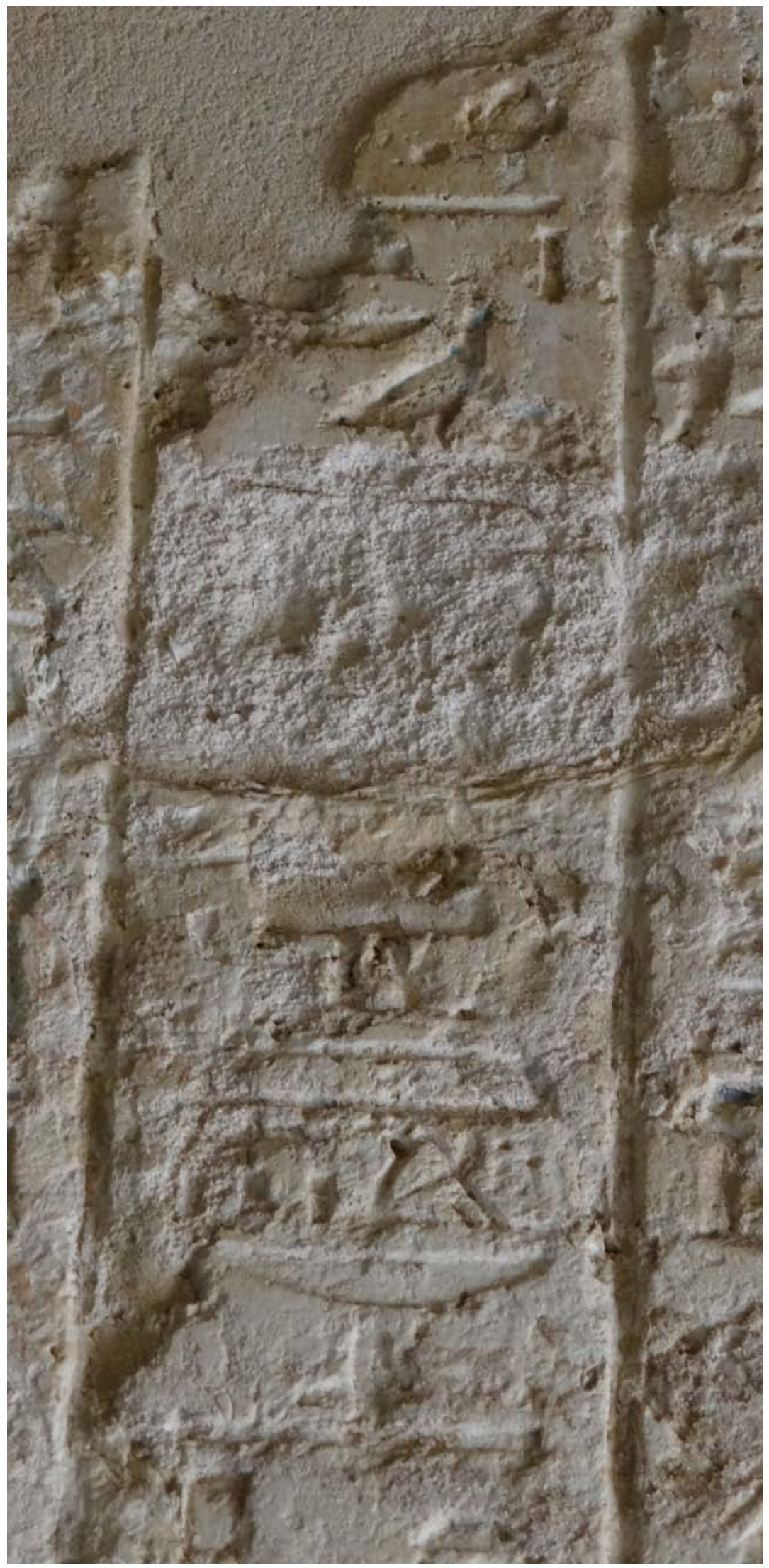

Fig. 2. Détail du milieu de la colonne 3 (cliché N. Gauthier, février 2015) 


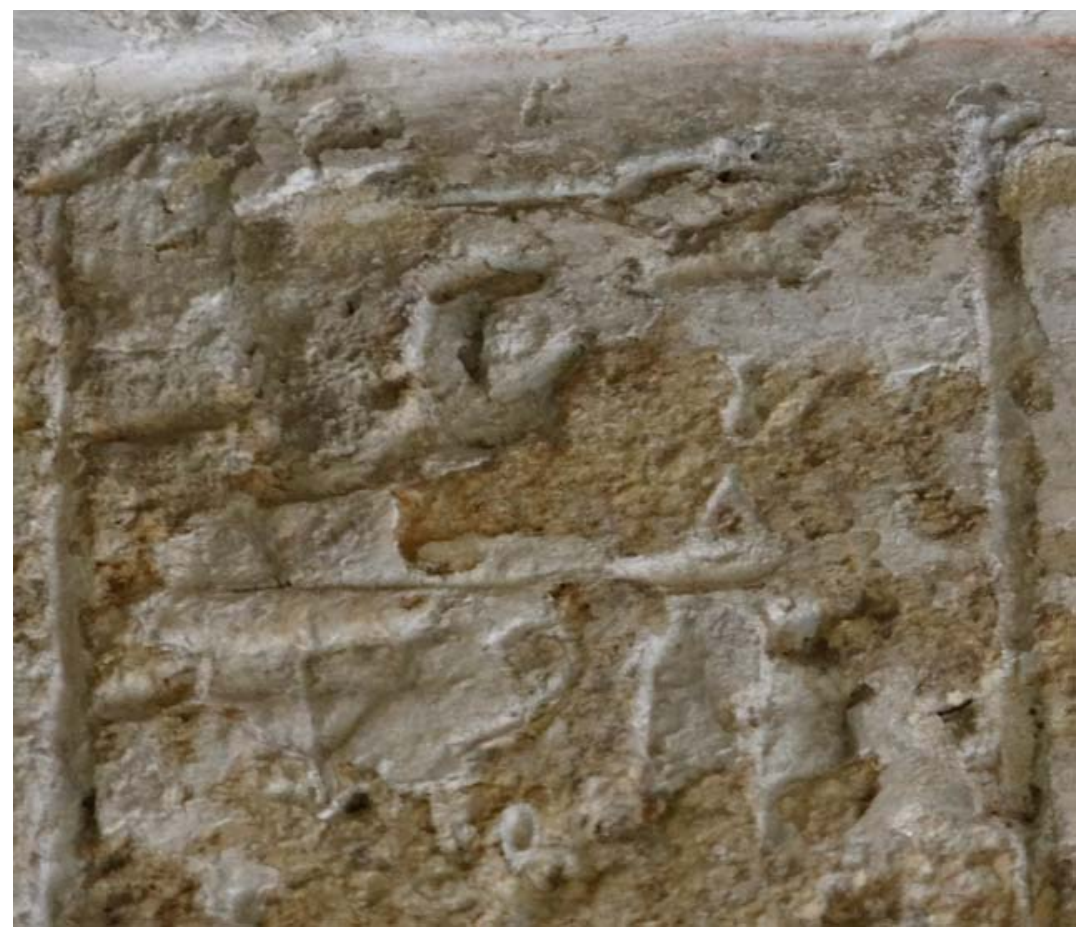

Fig. 3. Détail du haut de la colonne 7 (cliché N. Gauthier, février 2015) 